\title{
High efficacy of Sofosbuvir plus Simeprevir in a large cohort of Spanish cirrhotic patients infected with genotypes 1 and 4
}

Zoe Mariño ${ }^{1}$, Juan M. Pascasio-Acevedo ${ }^{2}$, Adolfo Gallego ${ }^{3}$, Moisés Diago ${ }^{4}$, Carme Baliellas ${ }^{5}$, Rosa Morillas $^{6}$, Martín Prieto ${ }^{7}$, José M. Moreno ${ }^{8}$, Gloria Sánchez-Antolín ${ }^{9}$, Mercedes Vergara ${ }^{10}$, Montserrat Forné ${ }^{1}$, Inmaculada Fernández ${ }^{12}$, María A. Castro ${ }^{13}$, Sonia Pascual ${ }^{14}$, Alexandra Gómez $^{15}$, Lluis Castells ${ }^{16}$, José L. Montero ${ }^{17}$, Javier Crespo ${ }^{18}$, José L. Calleja ${ }^{19}$, Javier GarcíaSamaniego $^{20}$, Jose A. Carrión ${ }^{21}$, Ana C. Arencibia ${ }^{22}$, Alejandro Blasco ${ }^{23}$, Carmen LópezNúñez ${ }^{24}$, Juan J. Sánchez-Ruano ${ }^{25}$, Francisco Gea-Rodríguez ${ }^{26}$, Álvaro Giráldez ${ }^{2}$, Joaquín Cabezas $^{18}$, Vanessa Hontangas ${ }^{7}$, Xavier Torras ${ }^{3}$, Jose Castellote ${ }^{5}$, Manuel Romero-Gómez ${ }^{27}$, Juan Turnes $^{28}$, Tomás de Artaza ${ }^{25}$, Isidoro Narváez ${ }^{29}$, Valentín Cuervas-Mons ${ }^{19}$, Xavier Forns ${ }^{1}$

\footnotetext{
${ }^{1}$ Liver Unit, Hospital Clinic, University of Barcelona, Institut d'Investigacions Biomèdiques August Pi i Sunyer (IDIBAPS) and Centro de Investigación Biomédica en Red de Enfermedades Hepáticas y Digestivas (CIBERehd), Barcelona, Spain

${ }^{2}$ Clinical Management Unit of Digestive Diseases, Hospital Universitario Virgen del Rocío, CIBERehd, Sevilla, Spain

${ }^{3}$ Liver Unit, Hospital Santa Creu i Sant Pau, CIBERehd, Barcelona, Spain

${ }^{4}$ Digestive Diseases, Hospital Universitario General, Valencia, Spain

${ }^{5}$ Liver Unit, Hospital Universitari Bellvitge-Instituto de Investigación Biomédica de Bellvitge (IDIBELL),

L'Hospitalet de Llobregat, Barcelona, Spain

${ }^{6}$ Liver Unit, Hospital Germans Trias i Pujol, CIBERehd, Badalona, Spain

${ }^{7}$ Liver Unit, Hospital Universitario La Fe, Valencia, Spain

${ }^{8}$ Department of Gastroenterology, Complejo Hospitalario Universitario de Albacete, Albacete, Spain

${ }^{9}$ Department of Gastroenterology, Hospital Universitario Río Hortega, Valladolid, Spain

${ }^{10}$ Liver Unit, Servei d'Aparell Digestiu, Parc Tauli Sabadell Hospital Universitari, Universitat Autónoma Barcelona, Sabadell, Spain

${ }^{11}$ Liver Unit, Hospital Universitario Mutua de Terrassa, Terrassa, Spain

${ }^{12}$ Liver Unit, Hospital Universitario 12 de Octubre, Madrid, Spain

${ }^{13}$ Internal Medicine, Grupo de Virología Clínica, Instituto Investigación Biomédica A Coruña (INIBIC)-Hospital Universitario A Coruña, A Coruña, Spain

${ }^{14}$ Liver Unit, Hospital General Universitario Alicante, CIBERehd, Alicante, Spain

${ }^{15}$ Liver Unit, Hospital Universitario Donostia, San Sebastian, Spain

${ }^{16}$ Liver Unit, Internal Medicine Department, Hospital Universitari Vall Hebron, CIBERehd, Barcelona, Spain

${ }^{17}$ Liver Unit, Hospital Universitario Reina Sofía, CIBERehd, Córdoba, Spain

${ }^{18}$ Gastroenterology and Hepatology Department, University Hospital Marqués de Valdecilla, IDIVAL, University of Cantabria, Santander, Spain

${ }^{19}$ Liver Unit, Hospital Universitario Puerta de Hierro, Universidad Autonoma de Madrid, CIBERehd, Madrid, Spain

${ }^{20}$ Liver Unit, Hospital Universitario La Paz, CIBERehd, IdiPAZ, Madrid, Spain

${ }^{21}$ Liver Section, Gastroenterology Department, Hospital del Mar, Universitat Autònoma de Barcelona, IMIM (Hospital del Mar Medical Research Institute), Barcelona, Spain

${ }^{22}$ Liver Unit, Hospital Universitario Nuestra Señora de Candelaria, Santa Cruz de Tenerife, Spain

${ }^{23}$ Digestive Diseases, Hospital de Sant Joan Despi Moises Broggi, Barcelona, Spain

${ }^{24}$ Digestive Diseases, Hospital Universitari Dr.Josep Trueta, Girona, Spain

${ }^{25}$ Digestive Diseases, Hospital Universitario de Toledo, Toledo, Spain

${ }^{26}$ Liver Unit, Hospital Ramón y Cajal, Madrid, Spain

${ }^{27}$ Liver Unit, Hospital Virgen de Valme, Sevilla, Spain

${ }^{28}$ Digestive Diseases, Complejo Hospitalario Universitario de Pontevedra and IISGS, Pontevedra, Spain

${ }^{29}$ Liver Unit, Hospital Infanta Cristina, Badajoz, Spain
} 


\begin{abstract}
Background and Aims. Hepatitis C (HCV) therapy with Sofosbuvir (SOF)/Simeprevir (SMV) in clinical trials and real-world clinical practice, showed high rates of sustained virological response (SVR) in non-cirrhotic genotype (GT)-1 and GT-4 patients. These results were slightly lower in cirrhotic patients. We investigated real-life effectiveness and safety of SOF/SMV with or without ribavirin (RBV) in a large cohort of cirrhotic patients.

Methods. This collaborative multicentre study included data from 968 patients with cirrhosis infected with HCV-GT1 or 4, treated with SOF/SMV \pm RBV in 30 centres across Spain between January-2014 and December-2015. Demographic, clinical, virological and safety data were analysed.

Results. Overall SVR was 92.3\%; the majority of patients were treated with RBV (62\%) for 12 weeks (92.4\%). No significant differences in SVR were observed between genotypes (GT1a:94.3\%; GT1b:91.7\%; GT4:91.1\%). Those patients with more advanced liver disease $(\mathrm{Child} \mathrm{B/C,MELD} \geq 10)$ or portal hypertension (platelet count $\leq 100 \times 10^{9} / \mathrm{L}$, transient elastography $\geq 21 \mathrm{Kpa}$ ) showed significantly lower SVR rates (84.4\%-91.9\%) than patients with less advanced liver disease $(93.8 \%-95.9 \%, P<.01$ in all cases). In the multivariate analysis, the use of RBV, female gender, baseline albumin $\geq 35 \mathrm{~g} / \mathrm{L}, \mathrm{MELD}<10$ and lack of exposure to a triple therapy regimen were independent predictors of SVR $(P<.05)$. Serious adverse events (SAEs) and SAE-associated discontinuation events occurred in $5.9 \%$ and $2.6 \%$.

Conclusions. In this large cohort of cirrhotic patients managed in the real-world setting in Spain, SOF/SMV \pm RBV yielded to excellent SVR rates, especially in patients with compensated liver cirrhosis. In addition, this combination showed to be safe, with low rates of SAEs and early discontinuations.
\end{abstract}

\title{
Keywords
}

Cirrhosis, Real-life cohort, Simeprevir, Sofosbuvir

\section{Abbreviations}

AE, adverse event; DAA, direct acting antivirals; GT, genotype; HCC, hepatocellular carcinoma; HCV, hepatitis C virus; HR, hazard ratio; IFN, interferon; KPa, kilopascal; MELD, model for end-stage-liver disease; PR, peg-IFN plus RBV; RAS, resistant-associated substitution; RBV, ribavirin; SAE, serious adverse event; SMV, Simeprevir; SOF, Sofosbuvir; SVR, sustained virological response; WBD, weight-based dose

\section{Key Points}

- The combination of SOF/SMV with or without RBV was highly effective (SVR 92.3\%) in real-life cirrhotic patients infected with genotypes 1 or 4 . Efficacy was reduced in patients with advanced liver disease and portal hypertension.

- The use of RBV, female gender, MELD $<10$, Albumin levels $\geq 35 \mathrm{~g} / \mathrm{L}$ and the lack of exposure to triple therapy were independent predictive factors of SVR.

- Safety was globally good and the rate of severe adverse events and/or premature discontinuations was rare.

- This antiviral option may still be considered as an alternative for compensated cirrhotic patients. 


\section{INTRODUCTION}

Chronic infection with the hepatitis C virus (HCV) constitutes a major global disease. ${ }^{1,2}$ Successful antiviral therapy leading to sustained virological response (SVR) significantly reduces the risk of HCVrelated cirrhosis and its complications ${ }^{3,4}$ and improves patients' quality of life. ${ }^{5}$ In Spain, the seroprevalence of $\mathrm{HCV}$ is around $2 \%$, being genotype (GT) $1 \mathrm{~b}$ the more frequent $(>70 \%) .{ }^{1}$

Until 2014, interferon (IFN)-based therapies were the only option for viral eradication. However, its use was contraindicated in decompensated liver disease ${ }^{6}$ and tolerability and efficacy were low in patients with cirrhosis. Triple therapy increased SVR rates to $70 \%-75 \%$ but further worsened safety profile. ${ }^{7}$ The main change in HCV therapy has been the introduction of oral direct acting antivirals (DAA). The first IFN-free DAA combination was the protease-inhibitor Simeprevir (SMV) plus the polymerase-inhibitor Sofosbuvir (SOF). This combination was first explored in a small phase-II trial ${ }^{8}$ including 167 GT1 patients, 41 of whom were cirrhotics. Treatment was well tolerated and overall SVR rate was $92.2 \%$. Based on these excellent results, SOF/SMV was approved by the FDA and EMA for the treatment of HCV. ${ }^{9-11}$ At that time, many difficult-to-treat patients with advanced liver disease, previous treatment failures or IFN contraindications underwent SOF/SMV. Since then, several studies of real-life have been published. ${ }^{12-16}$ One of the largest was the US-TARGET cohort, ${ }^{12}$ which included 491 cirrhotic patients (45\% with prior hepatic decompensation). Most of these patients (79.8\%) received SOF/SMV without RBV. Noteworthy, SVR rate was significantly lower in cirrhotic patients (80.5\%) than in non-cirrhotic patients $(89.5 \%)$. Similar results were observed in two phase-III clinical trials evaluating SOF/SMV without RBV, in which SVR was achieved in $83 \%$ of the cirrhotic patients ${ }^{17}$ compared to $97 \%$ in noncirrhotic patients. ${ }^{18}$

Despite other antiviral combinations have replaced SOF/SMV in many countries, in consonance with the recent international guidelines, ${ }^{19}$ there is still a high need for effective and affordable antiviral options in many areas of the world. Current access restrictions to new DAA also reinforce the need for updated data and evidence-based alternatives for $\mathrm{HCV}$ patients.

In Spain, the combination of SOF/SMV was extensively used in many referral centres. The existence of a national collaborative register allowed us to perform this study, in which we aimed at analysing the safety and efficacy of this combination in a large cohort of Spanish patients with well-defined liver cirrhosis, infected with GT1 and 4.

\section{PATIENTS AND METHODS}

\subsection{Study}

This was a retrospective, non-interventional, national, multicentre and collaborative study, evaluating the efficacy and safety of SOF/SMV with or without RBV for HCV-infected patients with cirrhosis in routine clinical practice in Spain. Data were collected through a National Registry (HEPA-C) and through a non-HEPA-C database from transplant centres.

\subsection{Patients}

The study recorded data of efficacy and safety from all cirrhotic patients infected with HCV GT1 (1a/1b) or 4, undergoing SOF/SMV with or without RBV between January 2014 and December 2015. Demographic, clinical, and virological data at baseline, treatment, and follow-up were collected. Cirrhosis was defined by transient elastography $\geq 14 \mathrm{kPa}$, liver biopsy, clinical evidence (such as presence of gastroesophageal varices or liver decompensation), or at least two ultrasonographical criteria (liver surface nodularity, enlarged spleen or portal vein diameter $>12 \mathrm{~mm}$ ). No other inclusion/exclusion criteria were applied. Patient follow-up was planned for 12 weeks after treatment interruption; safety data were 
reported for any patient receiving at least one dose of DAA. As it was a retrospective non-interventional analysis of common clinical practice, no specific informed consent was applied. This study was approved by the Research Ethics Committee of Hospital Clinic Barcelona.

\subsection{Treatment}

The duration of therapy (12 or 24 weeks) and the use and starting dose of RBV, was planned at the discretion of the treating physician, in accordance with the national and international recommendations at that time ${ }^{20,21}$ and the product labels. ${ }^{22-24}$ According to the Guidelines, patients with compensated cirrhosis should be treated for 12 weeks with daily weight-based dose (WBD) of RBV (1000 or $1200 \mathrm{mg}$ in patients $<75 \mathrm{~kg}$ or $\geq 75 \mathrm{~kg}$, respectively). ${ }^{19,20}$ Lower doses of RBV were considered by the authors as nonWBD. In those patients with contraindications or poor tolerance to RBV, treatment was extended for 24 weeks without RBV.

\subsection{Efficacy}

Sustained virological response (SVR) was defined as undetectable HCV-RNA 12 weeks after the end of treatment. Virological failure was defined as detectable HCV-RNA at any time during treatment or follow-up. HCV-RNA levels were determined using either the COBAS AmpliPrep ${ }^{\circledR} /$ COBAS TaqMan ${ }^{\circledR}$ (Roche Molecular Systems, Pleasanton, CA, USA; lower limit of detection [LLOD] $15 \mathrm{IU} / \mathrm{mL}$ ) or the m2000SP/m2000RT (Abbott Molecular, Des Moines, IL, USA; LLOD 12 IU/mL) real-time PCR-based assays, according to the different centres. Viral load measurements were reported at baseline and at 12 weeks after treatment completion.

\subsection{Safety}

All the adverse events (AE) reported by the investigators occurring during therapy or follow-up were registered retrospectively. The more usual events related with SOF, SMV or RBV were specified in order to simplify and unify the reports. These AE were: anaemia, asthenia, pruritus, hyperbilirubinaemia, dermatological events and digestive symptoms (diarrhoea, nausea or abdominal pain). Anaemia was defined as "nadir haemoglobin level $\leq 12 \mathrm{~g} / \mathrm{dL}$ during therapy"; severe anaemia was defined as "nadir haemoglobin level $\leq 10 \mathrm{~g} / \mathrm{dL}$ during therapy".

Severe adverse events (SAE) were defined as any life-threatening event (leading to hospital admission, prolonged hospital admission or death), or any event that was considered serious based on the treating physician's judgment. Incidence and reason for treatment discontinuation, liver decompensation and deaths were also recorded.

\subsection{Statistical analysis}

For descriptive purposes, quantitative variables are presented as mean, range and/or interquartile ranges (IQR), as appropriate. Categorical variables are presented as number and percentages. Efficacy and safety analyses were performed using the chi-squared test (categorical dicotomical variables), Student's $t$ test or Mann-Whitney test for comparisons between independent groups, at a significance level of .05. All the variables were tested first in univariate analysis, and included in a multivariate stepwise logistic-regression analysis if significant; selection of independent covariates by the regression model was based on a backward elimination procedure, retaining covariates with $P<.05$. Statistical analysis was performed with the SPSS® Statistics package, version 21 (IBM® Corporation, Somers, NY, USA). 


\section{RESULTS}

\subsection{Patient characteristics}

Thirty Spanish centres participated in this study, accounting for 1012 cirrhotic patients infected with GT1 or four treated with SOF/SMV \pm RBV. Out of them, $911(94.2 \%)$ were evaluable for the efficacy analysis and $898(92.8 \%)$ reported data for the safety profile (see Figure 1 for detailed flowchart).

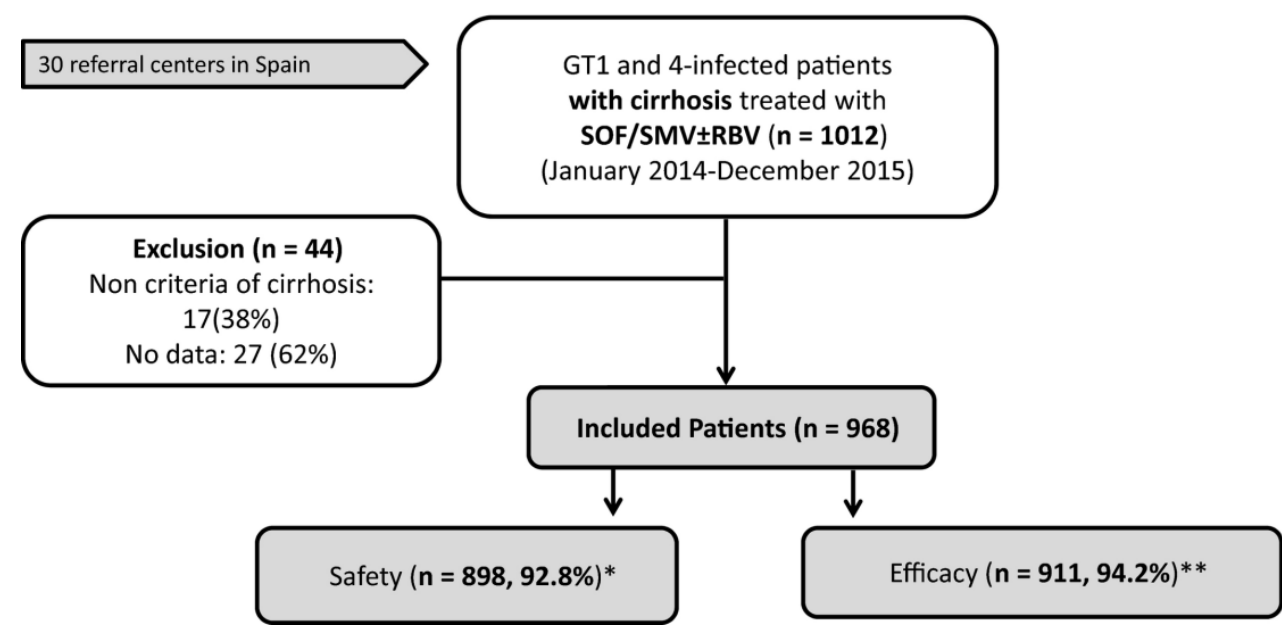

Figure 1. Flowchart of patients. (*) 70 patients had no data for safety purposes; (**) 57 patients had no data for efficacy purposes. GT, genotype; SOF, sofosbuvir; SMV, simeprevir

Regarding baseline features, $60.2 \%$ were males, median age was $60 \%$ and $69 \%$ were infected with subgenotype $1 \mathrm{~b}$. Importantly, $63 \%$ of included individuals were previous non-responders. One-third of patients had oesophageal varices and/or history of previous decompensation at baseline. The majority of patients were planned to receive RBV (61\%) and treated for 12 weeks (92.4\%). Detailed demographic and clinical characteristics are showed in Table 1. 
Table 1. Baseline Demographics of All Patients Who Started Treatment With Simeprevir Plus Sofosbuvir With or Without Ribavirin ( $\mathrm{n}=968)$

\begin{tabular}{|c|c|c|c|}
\hline & \\
\hline Variables & $\begin{array}{l}\text { Total Cohort } \\
(n=968)\end{array}$ & Variables & $\begin{array}{l}\text { Total Cohort } \\
(n=968)\end{array}$ \\
\hline & & Treatment planned duration (n, \%) & \\
\hline Gender (M) (n, \%) & $583(60.2)$ & $12 \mathrm{wk}$ & $894(92.4)$ \\
\hline Age (y) & $60(19-94)$ & $24 \mathrm{wk}$ & $39(4)$ \\
\hline Genotype (n, \%) & & Other durations & $9(0.9)$ \\
\hline $1 \mathrm{a}$ & $191(19.7)$ & NA & $26(2.7)$ \\
\hline $1 \mathrm{~b}$ & $671(69.3)$ & Planned use of RBV (n, \%) & \\
\hline $1(\mathrm{n} / \mathrm{t})$ & $23(2.4)$ & Yes & $590(61)$ \\
\hline 4 & $82(8.5)$ & No & $357(37)$ \\
\hline NA & $1(0.1)$ & NA & $20(2)$ \\
\hline Viral Load HCV-RNA $\left(\log _{10}\right)(\mathrm{UI} / \mathrm{mL})$ & $6.08(1.28-7.73)$ & & \\
\hline
\end{tabular}

Previous treatment experience (n, \%)

$\begin{array}{ll}\text { Yes } & 609(62.9) \\ \text { No } & 355(36.7) \\ \text { NA } & 4(0.4) \\ \begin{array}{l}\text { Previous triple therapy (TT) (protease- } \\ \text { inhibitor+Peg/RBV) }\end{array} & 119(12.3) \\ \text { Diagnosis of Liver Cirrhosis } & \\ \text { Elastographic criteria } & 326(33.7) \\ \text { Ultrasound Criteria } & 60(6.2) \\ \text { Clinical Criteria } & 179(18.5) \\ \text { More than 1 criteria } & 383(39.6) \\ \text { Transient Elastography (Kpa) } & \\ \text { TE } \geq 21 \text { Kpa (n, \%) } & 22(4-91)[P \\ \text { Child-Pugh score (n, \%) } & 16.8-33.3] \\ \text { A } & 393(40.6) \\ \text { B } & 738(76.2) \\ \text { C } & 159(16.4) \\ \text { NA } & 8(1) \\ \text { a } & 63(6.5)\end{array}$

MELD score (median, range); IQR [ $\mathrm{P}_{25^{-}} 8$ (7-26); IQR $\left.\mathrm{P}_{75}\right]^{\mathrm{b}} \quad\left[\mathrm{P}_{25}-\mathrm{P}_{75}: 7-10\right]$ MELD score $\geq 10(\mathrm{n}, \%) \quad 260(26.9)$

Any previous Liver Decompensation (n, $\%)$

$\begin{array}{ll}\text { Ascites } & 135(13.9) \\ \text { Hepatic encephalopathy } & 12(1.2) \\ \text { Variceal Bleeding } & 43(4.4) \\ \text { More than 1 decompensation } & 75(7.6) \\ \text { Not especified } & 32(3.3) \\ \text { Oesophageal or gastric varices at } & 320(33.1) \\ \text { baseline (n, \%) } & \\ \text { Platelets count }\left(10^{9} / \mathrm{L}\right) & 98(12-647) \\ \text { Platelet count } \leq 100(\mathrm{n}, \%) & 483(49.9) \\ \text { Haemoglobin }(\mathrm{g} / \mathrm{dL}) & 14(7.3-19) \\ \text { ALT }(\mathrm{UI} / \mathrm{L}) & 71(5-513) \\ \text { Bilirubin }(\mathrm{mg} / \mathrm{dL}) & 0.99(0.20-6) \\ \text { Albumin }(\mathrm{g} / \mathrm{L}) & 39(22-52) \\ \text { Albumin level }<35 \mathrm{~g} / \mathrm{L}(\mathrm{n}, \%) & 198(20.5)\end{array}$

Table 1. Baseline Demographics of All Patients Who Started Treatment With Simeprevir Plus Sofosbuvir With or Without Ribavirin (n=968)

M, male; F, female; NA, not available; HCV, hepatitis C virus; TE, transient elastography; KPa, kilopascals; MELD, Model for End-Stage Liver Disease; IQR, interquartile range; RBV, ribavirin; n/t, not typable; Peg, pegylated.

Quantitative variables are presented as mean and range and/or interquartile ranges (IQR, $\left.\mathrm{P}_{25}-\mathrm{P}_{75}\right)$ as appropriate. Categorical variables are presented as number and percentages $(n, \%)$. Data refer to the whole population receiving at least one dose of the study medication. For efficacy purposes, only 911 patients could be analysed.

${ }^{a}$ Available in 705 patients.

${ }^{\mathrm{b}}$ Available in 857 patients.

${ }^{c}$ Available in 737 patients. 


\subsection{Efficacy analysis}

Virological evaluation at follow-up 12 was available in 911 (94.2\%) patients. Overall, the crude SVR rate was $92.3 \%$. No significant differences of efficacy were detected between genotypes $(92.4 \%$ in GT1 vs $91.1 \%$ in GT4, $P=.4$ ), subgenotypes ( $94.3 \%$ in GT1a vs $91.7 \%$ in GT1b, $P=.15$ ) or treatment duration (92.3\% for 12 weeks vs $96.7 \%$ for 24 weeks, $P=.32$ ). Similar SVR rates were also obtained in naive and previously non-responders to dual therapy (PR; 94.2\% vs 91.2\%, $P=.07$ ). However, the subgroup of patients who had failed to previous triple therapy $(n=115)$ had lower SVR $(86.1 \% ; P=.03$, compared to PR). SVR rates were higher in female patients compared to males $(94.4 \%$ vs $90.9 \%, P=.03$; Figure $2 \mathrm{~A})$.
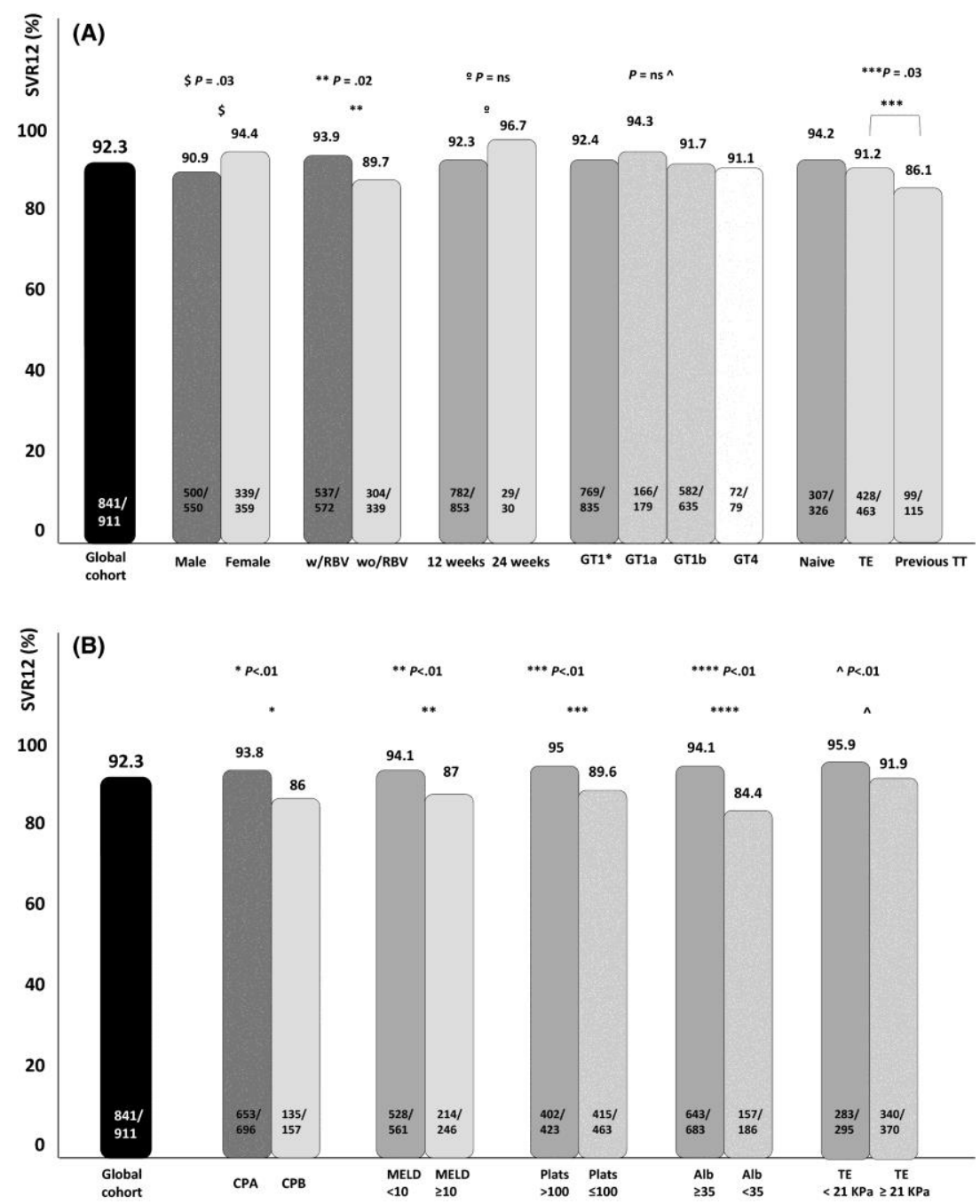

Figure 2 (A) SVR12 Rates Among Patients With Available Outcomes according to baseline and treatment characteristics. $(\wedge)$ Refers to GT1 vs GT4 y GT1a vs GT1b $(P=$ ns). (B) SVR12 Rates Among Patients With Available Outcomes according to liver disease severity. W/RBV, with Ribavirin; wo/RBV, without Ribavirin; GT, genotype; wk, weeks; TE, treatment experienced; TT, triple therapy with a protease-inhibitor; CPA, Child-Pugh A score; CPB/C, Child-Pugh B/C; MELD, model for end-stage liver disease; plats, platelets; Alb, albumin; TE, transient elastography; Kpa, kilopascals 
Patients with more advanced liver disease (Child-Pugh score B/C, MELD score $\geq 10$, or indirect markers of portal hypertension such as platelets $\leq 100000$ or transient elastography $\geq 21 \mathrm{Kpa})^{25}$ presented significantly lower SVR rates (84.4\%-91.9\%) when compared to patients with less advanced liver disease (Child-Pugh A, MELD score<10, no markers of portal hypertension; 93.8\%-95.9\%; $P<.05$; Figure 2B).

The majority of patients (62.8\%) received concomitant RBV as part of their treatment, independently of the gender $(P=.14)$. In most of them (59.7\%), RBV was dosed according to weight. Overall, the use of RBV was associated with significantly higher SVR rates: $93.9 \%$ vs $89.7 \%$ in those without RBV $(P=.02)$. However, we found no differences neither between patients receiving weight-based dose (WBD) vs nonWBD of RBV (93.4\% vs 94\%, $P=.47$ ), nor between the different subgenotypes (depicted in Figure 3). RBV was similarly used irrespectively of the severity of cirrhosis $(66.3 \%$ in Child A vs $63.7 \%$ in Child $\mathrm{B} / \mathrm{C}, P=\mathrm{ns}$ ). Noteworthy, in patients with advanced liver disease (Child-Pugh $\mathrm{B} / \mathrm{C}, \mathrm{n}=157$ ) treated without RBV, SVR decreased to $82.5 \%$ (vs $88 \%$ if RBV was added), although this difference did not reach statistical significance $(P=.23)$.

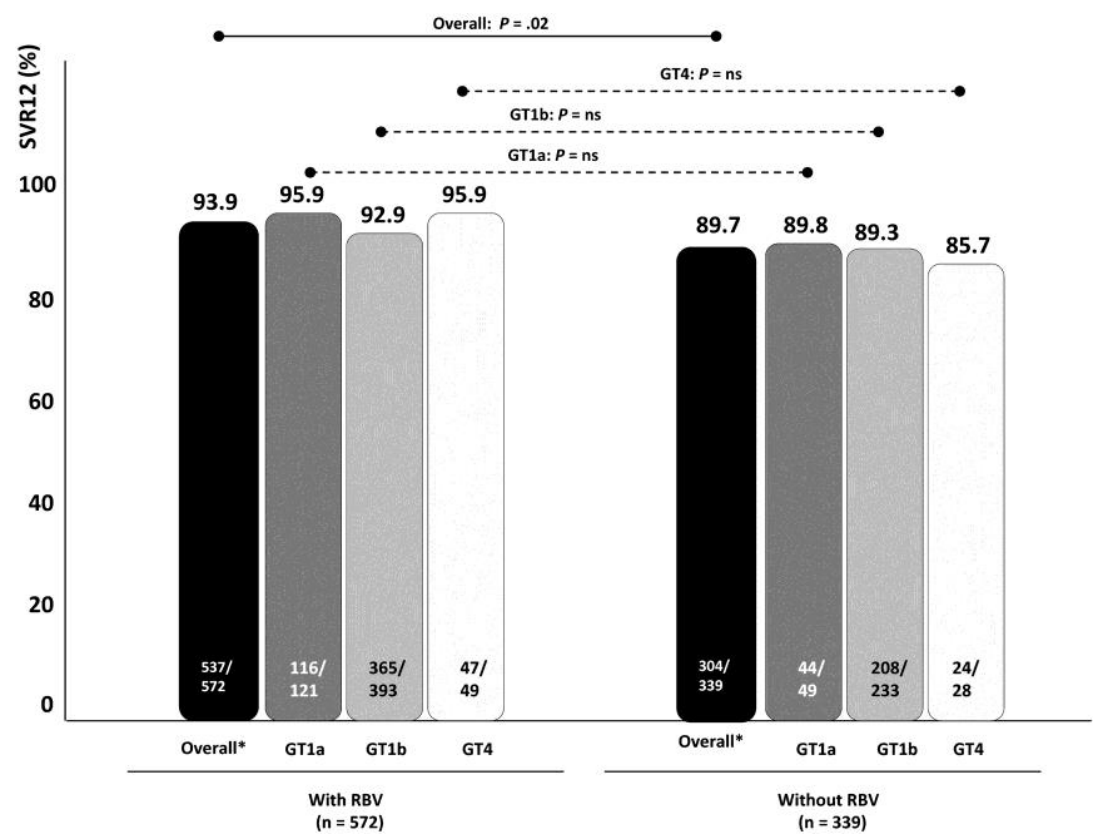

Figure 3 SVR12 Rates according to the use of RBV in the different viral subgenotypes. (*) Subgenotype was not available in 21 GT1 patients which were not included in the bars

In the multivariate analysis, the use of RBV (HR 1.93 [1.13-3.28], $P=.015$ ) and female gender (HR 2.15 [1.19-3.89], $P=.011$ ) were independent predictors of SVR, whereas a MELD score $\geq 10$ (HR 0.51 [0.28-0.91], $P=.024)$, baseline albumin levels $<35 \mathrm{~g} / \mathrm{L}$ (HR 0.43 [0.23-0.78], $P=.006)$ and previous failure to triple therapy (HR 0.33 [0.17-0.64], $P=.001$ ), were inversely associated with the achievement of SVR (Table 2). 
Table 2. Univariate and multivariate predictors of SVR in the efficacy cohort $(n=911)$

\begin{tabular}{|c|c|c|c|c|c|c|}
\hline Variables & $\begin{array}{l}\text { SVR } \\
(n=841)\end{array}$ & $\begin{array}{l}\text { Non SVR } \\
(\mathrm{n}=70)\end{array}$ & $\begin{array}{l}P^{\mathrm{a}}(\text { Univariate } \\
\text { analysis) }\end{array}$ & $\begin{array}{l}\mathrm{HR}(95 \% \mathrm{CI}) \\
\text { Univariate }\end{array}$ & $\begin{array}{l}P^{\mathrm{b}} \text { (Multivariate } \\
\text { analysis) }\end{array}$ & $\begin{array}{l}\text { HR }(95 \% \mathrm{CI}) \\
\text { Multivariate }\end{array}$ \\
\hline Age (y) & $60(53-68)$ & $58.9(51-66)$ & .24 & $1.01(0.99-1.04)$ & & \\
\hline Gender (F) (vs M) & $\begin{array}{l}339 / 839 \\
(40.4 \%)\end{array}$ & $\begin{array}{l}20 / 70 \\
(28.6 \%)\end{array}$ & .05 & $1.69(0.99-2.89)$ & .011 & $2.15(1.19-3.89)$ \\
\hline Use of RBV (n, \%) & $\begin{array}{l}563 / 822 \\
(65.2 \%)\end{array}$ & $\begin{array}{l}35 / 69 \\
(50.7 \%)\end{array}$ & .02 & $1.76(1.08-2.88)$ & .015 & $1.93(1.13-3.28)$ \\
\hline GT1b (vs GT1a) & $\begin{array}{l}592 / 761 \\
(77.8 \%)\end{array}$ & $53 / 63(84 \%)$ & .24 & $0.66(0.32-1.32)$ & & \\
\hline $\begin{array}{l}\text { Child-Pugh score B/C } \\
\text { (vs CPA) }\end{array}$ & $\begin{array}{l}135 / 788 \\
(17.1 \%)\end{array}$ & $\begin{array}{l}22 / 65 \\
(33.8 \%)\end{array}$ & .001 & $0.40(0.23-0-69)$ & & \\
\hline $\operatorname{MELD} \geq 10(\mathrm{n}, \%)^{\mathrm{c}}$ & $\begin{array}{l}214 / 742 \\
(28.8 \%)\end{array}$ & $\begin{array}{l}32 / 65 \\
(49.2 \%)\end{array}$ & .001 & $0.42(0.25-0.69)$ & .024 & $0.51(0.28-0.91)$ \\
\hline $\begin{array}{l}\text { Transient elastography } \\
(\mathrm{KPa})\end{array}$ & $\begin{array}{l}21.8(16.6- \\
32.8)\end{array}$ & $\begin{array}{l}26.8(19.6- \\
35.4)\end{array}$ & .16 & $0.98(0.96-1.00)$ & & \\
\hline $\begin{array}{l}\text { Baseline Oesophageal } \\
\text { varices }\end{array}$ & $\begin{array}{l}280 / 644 \\
(43.5 \%)\end{array}$ & $\begin{array}{l}25 / 55 \\
(45.5 \%)\end{array}$ & .77 & $0.92(0.53-1.60)$ & & \\
\hline Platelets $\left(10^{9} / \mathrm{mL}\right)$ & $\begin{array}{l}100(68- \\
141)\end{array}$ & $82(59-113)$ & .04 & $1.01(1.00-1.01)$ & & \\
\hline $\begin{array}{l}\text { Platelet count } \leq 100 \\
\left(10^{9} / \mathrm{mL}\right)\end{array}$ & $\begin{array}{l}415 / 817 \\
(50.8 \%)\end{array}$ & $\begin{array}{l}48 / 69 \\
(69.6 \%)\end{array}$ & .003 & $0.45(0.26-0.76)$ & & \\
\hline Albumin (g/L) & $\begin{array}{l}39.9(35.9- \\
42.3)\end{array}$ & $37(32-40.7)$ & $<.001$ & $1.09(1.04-1.14)$ & & \\
\hline $\begin{array}{l}\text { Albumin levels <35 } \\
(\mathrm{g} / \mathrm{L})\end{array}$ & $\begin{array}{l}157 / 800 \\
(19.6 \%)\end{array}$ & $29 / 69(42 \%)$ & $<.001$ & $0.34(0.20-0.56)$ & .006 & $0.43(0.23-0.78)$ \\
\hline $\begin{array}{l}\text { Previous failure to TT } \\
(\mathrm{n}, \%)\end{array}$ & $\begin{array}{l}99 / 841 \\
(11.8 \%)\end{array}$ & $\begin{array}{l}16 / 70 \\
(22.9 \%)\end{array}$ & .009 & $0.45(0.24-0.81)$ & .001 & $0.33(0.17-0.64)$ \\
\hline
\end{tabular}

M, male; GT, genotype; MELD, model for end-stage liver disease; KPa, kilopascals; SVR, sustained virological response; OR, odds ratio; $\mathrm{CI}$, confidence interval.

Statistically significant values are represented in bold numbers.

${ }^{a}$ Univariate analysis (as appropriate): $T$ Student/ $U$ Mann Whitney for continuous variables; Chi square in categorical variables

${ }^{\mathrm{b}}$ Multivariate analysis: logistic regression stepwise.

${ }^{\mathrm{c}}$ MELD score also remained in the uni and multivariate analysis when considered as a continuous variable.

\subsection{Safety analysis}

Safety and tolerability was available in 898 patients $(92.8 \%)$. Overall, safety was good despite the high incidence of adverse events (AE; 48.6\%; Table 3). Noteworthy, most of the AE were mild (91\%) and mainly occurred in patients receiving RBV (72.4\%; $P<.01)$. Some AE (2.8\%), were reported as not related with antiviral treatment. 
Table 3. Safety profile

\begin{tabular}{|c|c|}
\hline Safety Events & Cohort $(n=898)$ \\
\hline Any Adverse Events (AE) (n, \%) & $436(48.6 \%)$ \\
\hline Treatment related $\mathrm{AE}$ & $411(45.8 \%)$ \\
\hline Non-treatment-related $\mathrm{AE}$ & $25(2.8 \%)$ \\
\hline Unknown association & $28(3.1 \%)$ \\
\hline \multicolumn{2}{|l|}{$\mathrm{AE}$ according to treatment $\operatorname{arm}(\mathrm{n}, \%)$} \\
\hline RBV-cohort $(\mathrm{n}=527)$ & $312(72.4 \%)$ \\
\hline Non RBV-cohort $(n=323)$ & $119(27.6 \%)$ \\
\hline \multicolumn{2}{|l|}{ More frequent $\mathrm{AE}$ (global incidence $\geq 10 \%)^{\mathrm{a}}$} \\
\hline Anaemia (nadir $\mathrm{Hb} \leq 12 \mathrm{~g} / \mathrm{dL}$ ) & $403(44.9 \%)$ \\
\hline Asthenia & $294(32.8 \%)$ \\
\hline Dermathologic events & $136(15.2 \%)$ \\
\hline Hyperbilirrubinaemia & $308(34.3 \%)$ \\
\hline \multicolumn{2}{|l|}{ Anaemia according to treatment arm (n, \%) } \\
\hline \multicolumn{2}{|l|}{$R B V$-group } \\
\hline Anaemia (nadir $\mathrm{Hb} \leq 12 \mathrm{~g} / \mathrm{dL}$ ) & $294(54 \%)$ \\
\hline Severe anaemia $(\mathrm{Hb} \leq 10 \mathrm{~g} / \mathrm{dL})$ & $73(13.4 \%)$ \\
\hline Nadir level of $\mathrm{Hb}(\mathrm{g} / \mathrm{dL})$ & $11.9(6.3-17.5)$ \\
\hline \multicolumn{2}{|l|}{ Non $R B V$-group } \\
\hline Anaemia (nadir $\mathrm{Hb} \leq 12 \mathrm{~g} / \mathrm{dL}$ ) & $105(32.9 \%)$ \\
\hline Severe anaemia $(\mathrm{Hb} \leq 10 \mathrm{~g} / \mathrm{dL})(\mathrm{n}, \%)$ & $42(13.2 \%)$ \\
\hline Nadir level of $\mathrm{Hb}(\mathrm{g} / \mathrm{dL})$ & $13.2(6.5-18.4)$ \\
\hline \multicolumn{2}{|l|}{ Management of anaemia } \\
\hline RBV dose reduction/discontinuation & $109(35.4 \%) / 16(5.2 \%)$ \\
\hline Use of EPO/Blood transfusion & $15(4 \%) / 21(5.2 \%)$ \\
\hline Serious Adverse Events $(\mathrm{SAE})^{\mathrm{b}}(\mathrm{n}, \%)$ & $53(5.9 \%)$ \\
\hline Early discontinuation because of $\mathrm{AE}^{\mathrm{c}}(\mathrm{n}, \%)$ & $23(2.6 \%)$ \\
\hline Liver decompensation $^{\mathrm{d}}$ & $56(6.2 \%)$ \\
\hline Deaths $^{\mathrm{e}}$ & $31(3.5 \%)$ \\
\hline Liver-related causes & $18(58.1 \%)$ \\
\hline Non-liver-related causes & $9(29 \%)$ \\
\hline Unknown & $4(12.9 \%)$ \\
\hline
\end{tabular}

\footnotetext{
${ }^{a}$ Other $\mathrm{AE}$ with an incidence <10\%: insomnia (7.6\%), headache (6.7\%), digestive events $(6.1 \%)$, flu-syndrome (1.9\%), renal impairment (1.6\%), infections (5.4\%).

${ }^{b}$ Serious Adverse events: severe allergic reaction $(n=1)$, suicide $(n=1)$, de novo HCC $(n=2)$, pneumonia $(n=1)$, unknown cause of intratreatment non-hepatic exitus $(n=2)$, neurologic disorder $(n=1)$, medular aplasia $(n=1)$, de novo liver decompensation $(n=24)$, liver function impairment $(\mathrm{n}=2)$, myositis $(\mathrm{n}=1)$, unknown $(\mathrm{n}=17)$.

${ }^{c}$ Early discontinuation: severe allergic reaction $(n=1)$, aplasia medular $(n=1)$, on-treatment virological failure $(n=1)$, de novo HCC $(n=1)$, neurologic disorder $(n=1)$, liver decompensation $(n=6)$, liver function impairment $(n=4)$, suicide $(n=1)$, unknown cause of intratreatment non-hepatic exitus $(\mathrm{n}=2)$, tiredness $(\mathrm{n}=1)$, pneumonia $(\mathrm{n}=1)$, pruritus/rash $(\mathrm{n}=2)$, liver transplantation $(\mathrm{n}=1)$.

${ }^{\mathrm{d}}$ Includes de novo decompensation and impairment of a previous stable situation.

${ }^{\mathrm{e}}$ Deaths: pneumonia leading to sepsis $(\mathrm{n}=1)$, suicide $(\mathrm{n}=1)$, lymphoma $(\mathrm{n}=1)$, liver disease progression $(\mathrm{n}=12)$, HCC progression $(\mathrm{n}=5)$, unknown $(\mathrm{n}=11)$.
} 
In the overall population, the most common $\mathrm{AE}(\geq 10 \%)$ were: anaemia (44.9\%), asthenia (32.7\%), hyperbilirrubinaemia (34.3\%) and dermatological events (15.2\%). As expected, anaemia (nadir haemoglobin $\leq 12 \mathrm{~g} / \mathrm{dL}$ ) was significantly more frequent in patients receiving RBV (54\% vs $32.9 \%$ in those without RBV, $P<.01$ ). Severe anaemia (nadir haemoglobin $\leq 10 \mathrm{~g} / \mathrm{dL}$ ) was reported in 115 patients (12.8\%); $63.5 \%$ of them received RBV. Anaemia was mainly managed by RBV dose reduction (35.4\%) or discontinuation $(5.2 \%)$. Importantly, the need for erythropoietin or blood transfusion was very uncommon ( $4 \%$ and $5.2 \%$ respectively).

Severe adverse events (SAE) were registered in 53 patients (5.9\%). Early treatment discontinuation of antiviral therapy occurred in a minority of cases $(n=23,2.6 \%)$. Decompensation of liver cirrhosis was reported in 56 patients $(6.2 \%)$ and included both de novo events $(n=23)$, as well as worsening of a previous stable condition $(\mathrm{n}=33)$. Thirty-one patients died $(3.5 \%)$ during the period of the study, mainly (58\%) because of liver-related causes. Details of SAE are depicted in Table 3.

Hepatocellular carcinoma (HCC) recurrence was reported in nine of 33 (27.3\%) patients with documented HCC at the time of treatment initiation. Moreover, 18 (1.9\%) patients were diagnosed with HCC during the study period. HCC progression was the cause of death in five cases. However, this study was not conducted to assess recurrence or incidence of HCC, and these data must be considered with caution.

\section{DISCUSSION}

Real-world clinical experience is relevant to validate efficacy and safety of drugs after controlled clinical trials and approval by Health Authorities. Indeed, efficacy in real-life is often worse than reported, as patients are more heterogeneous, physicians may face unusual circumstances, and some unexpected safety concerns may also be raised when treating complex patients.

Based on the favourable results of SOF/SMV in clinical trials, ${ }^{17,18}$ many real-life cohort studies were published after drug approval in $2014 .{ }^{12,15,16,26}$ Safety profile was good ${ }^{27}$ whereas efficacy was reported to be excellent in non-cirrhotics (SVR 92\%-94\%) but slightly lower in patients with cirrhosis (SVR 82\%$85 \%){ }^{12,28}$ For this reason, we aimed at assessing the efficacy and safety of SOF/SMV in our cohort of Spanish cirrhotic patients. This is, at the best of our knowledge, the largest cohort of cirrhotic patients treated with this combination.

SOF/SMV treatment with or without RBV achieved overall SVR rates of $92.3 \%$ in our cohort, which is largely enriched by patients with advanced liver disease and other unfavourable predictors of antiviral response: up to one-third had a history of previous liver decompensation and/or oesophageal varices at baseline, and the proportion of patients with previous treatment failure to PR or triple therapy was remarkable. This may also explain the frequent use of RBV, which was indicated in two-thirds of patients. As expected, SVR rates were slightly lower in patients with more advanced liver disease (ChildPugh B/C: SVR 86\%; MELD $\geq 10$ : SVR 87\%; Albumin levels $<35$ g/L: SVR 84.4\%) and in patients who had failed to triple therapy (SVR 86.1\%). At multivariate analysis, the use of RBV, female gender, MELD score $<10$, baseline albumin levels $\geq 35 \mathrm{~g} / \mathrm{L}$ and no previous exposure to triple therapy were identified as independent predictors for SVR.

It is important to notice that the overall efficacy of this regimen in our cohort is clearly higher than the efficacy published in other large series. ${ }^{28}$ There are several potential explanations for these results. Firstly, a more frequent use of RBV (with potential additive antiviral effect), which might be important in difficult-to-treat patients. Noteworthy, this beneficial effect was independent of the starting dose of RBV. As stated above, RBV was used in almost $63 \%$ of patients, compared to only $22 \%$ in the TARGET cohort, ${ }^{12}$ where RBV was not associated with improved SVR rates. Conversely, the STIly Italian multicentric study ${ }^{29}$ reported high SVR rates in a cohort of 263 cirrhotic patients treated with SOF/SMV (82\% with RBV). Although no differences in efficacy were described between those receiving RBV or not $(91 \%$ vs $96 \%)$, patients treated with RBV presented a more advanced liver disease, probably 
impacting global results. Another multicentric study including 270 GT1 compensated cirrhotics treated for 12 weeks with SOF/SMV plus $\mathrm{RBV}^{30}$ reported excellent efficacy (SVR 95.9\%) and tolerability $(<2 \%$ $\mathrm{SAE},<6 \%$ discontinuation). On the other hand, supporting the usefulness of RBV in cirrhotic patients, the OPTIMIST 2 phase-III trial ${ }^{17}$ reported efficacy rates of only $83 \%$ in a cohort of 103 compensated cirrhotic patients treated for 12 weeks with SOF/SMV without RBV. A second explanation for the good results obtained in our cohort is the lower prevalence of GT1a-infected patients in Spain, and the reduced European prevalence of Q80K mutation in GT1a compared to USA. ${ }^{31,32}$ Although the negative impact of Q80K resistant-associated substitution (RAS) in GT1a patients undergoing SOF/SMV is not as evident as in those treated with PR plus SMV, ${ }^{33,34}$ SVR rates were lower in patients harbouring this RAS both in the $\operatorname{COSMOS}^{8}$ and OPTIMIST-II ${ }^{30}$ trials. This is in line with the reduced SVR rates observed in patients previously exposed to a protease-inhibitor as part of triple therapy. We did not find a difference in efficacy between GT1a and GT1b patients, but this may be explained by the wide use of RBV. A third issue regarding the high efficacy of SOF/SMV in our population is the fact that patients were treated by hepatologists in referral centres rather than in community-based practice; this may result in greater treatment adherence, better management of $\mathrm{AE}$ and, secondarily, in higher response rates. ${ }^{35}$

Safety was good in our cohort of cirrhotic patients. As expected, AE were more frequent in patients receiving RBV. However and similarly to previous reported data, only a few proportion of patients presented SAE or required premature treatment interruption. Anaemia was easily managed mainly by dose reductions of RBV. Considering the warning FDA/EMA reports regarding the use of proteaseinhibitors in advanced cirrhosis, treating physicians were prone to assess the liver-related SAE. In our experience, SAE were rare $(5.9 \%)$ and decompensation rates low $(6.2 \%)$. The data can be considered within the expected range of events in patients not undergoing therapy, although a control group would be necessary to support this statement. Nevertheless, our study reinforces that the use of SOF/SMV in compensated cirrhosis is safe.

This study has the inherent limitations related to its observational, multicentric and real-world design. This is more evident for the safety evaluation, since data were not captured prospectively and might be incomplete. A potential bias for using RBV by the treating physicians cannot be excluded. Nevertheless, this large real-life experience gives an important insight into the effectiveness and safety of SOF/SMV for GT1 and GT4 cirrhotic patients.

The American Association Guidelines for treatment of $\mathrm{HCV}^{36}$ consider SOF/SMV as an option for non-cirrhotic GT1 patients; however, SOF/SMV remains as a secondary alternative in those with compensated cirrhosis and should be administered for 24 weeks. In the European Guidelines in 2016, ${ }^{19}$ SOF/SMV is not considered an optimal alternative for GT1 patients anymore. In both cases, the decision is supported by the slightly better results of other DAA (Sofosbuvir/ledipasvir, Sofosbuvir/Velpatasvir, Paritaprevir/Ombitasvir/ritonavir with Dasabuvir, or Grazoprevir/Elbasvir) that emerged afterwards in 2015-2016 improving antiviral efficacy and minimizing the need for RBV. ${ }^{19,36}$ However, based on our results, SOF/SMV (with RBV addition) should be still considered an effective and safe therapy for GT1 and GT4 cirrhotic patients, if other combinations are not available or affordable. This is the case for many countries in Central and South America, as well as other regions of the world such as Africa and Asia.

Moreover, the use of SMV in such a large cohort of cirrhotic patients is important to reinforce the safety profile of protease-inhibitors in this setting, where other agents from this class (voxilaprevir, glecaprevir) will be widely used in the near future. 


\section{CONFLICT OF INTERESTS}

ZM: Speaker for Abbvie, Gilead, Janssen; advisory board for Gilead, BMS. JMP: Consultant and speaker for Gilead, Abbvie, Janssen, BMS, MSD. AG: Speaker for Gilead, Janssen, Bristol. CB: Speaker for Abbvie, Janssen. RMaM: Speaker for Janssen, Merck, BMS, Gilead, AbbVie; Advisory Board for Merck, BMS, Gilead, AbbVie. MV: Speaker for Janssen, Gilead, Abbvie. IF: Speaker and consultant for Janssen, Abbvie, MSD, Bristol, Gilead. SP: Speaker for Gilead, Bayer, MSD. JLC: Speaker and consultant: MSD, Gilead, Abbvie, Janssen. JGS: Grant Support from Gilead. Speaker and consultant: Abbvie, BMS, Gilead, Janssen. JJSR: Speaker for Gilead, Abbvie; Advisory Board for Gilead, MSD. FGR: Speaker for BMS, Gilead, Abbvie; Advisory Board for Gilead, Abbvie. AG: Speaker for Abbvie, Gilead, Gore. VH: Speaker for Janssen, Gilead, Abbvie. XT: Speaker for Janssen, Gilead, MSD, Abbvie; advisory board for Gilead, Abbvie. JC: Speaker for Abbvie, Gilead. XF: Unrestricted Grant Support from Abbvie. Advisor for Abbvie, Gilead, Janssen. JT: speaker and advisory board for Abbvie, Gilead, Janssen, BMS; speaker for MSD. TA: Speaker for Janssen, AbbVie. IN: Speaker for Janssen, Gilead; advisor for Abbvie. MD, MP, JMM, GSA, MF, MAC, AG, LC, JLM, JC, JAC, ACA, AB, CL, JC, MR, VCM: None declared.

\section{REFERENCES}

1. Messina JP, Humphreys I, Flaxman A, et al. Global distribution and prevalence of hepatitis C virus genotypes. Hepatology. 2015;61:77-87.

2. World Health Assoc. Hepatitis C: WHO Fact Sheet 2016.

http://www.who.int/mediacentre/factsheets/fs164/en/. Accessed April 15, 2017.

3. van der Meer AJ, Heathcote EJ, Janssen HL. Association between sustained virological and advanced hepatic fibrosis. JAMA. 2012;308:2584-2593.

4. Backus LI, Belperio PS. Impact of sustained virologic response on all-cause mortality. Hepatology. 2013;58:1508-1510.

5. Younossi ZM, Stepanova M, Sulkowski M, et al. Ribavirin-free regimen with Sofosbuvir and Velpatasvir is associated with high efficacy and improvement of patient-reported outcomes in patients with genotypes 2 and 3 chronic hepatitis c: results from astral- 2 and -3 clinical trials. Clin Infect Dis. 2016;63:1042-1048.

6. Carrión JA, Martínez-Bauer E, Crespo G, et al. Antiviral therapy increases the risk of bacterial infections in $\mathrm{HCV}$-infected cirrhotic patients awaiting liver transplantation: a retrospective study. J Hepatol. 2009;50:719-728.

7. Mangia A, Graham RF, Berg CP, et al. Efficacy and safety profile of boceprevir- or telaprevirbased triple therapy or dual peginterferon alfa- $2 a$ or alfa- $2 b$ plus ribavirin therapy in chronic hepatitis C: the real-world PegBase observational study. Ann Gastroenterol. 2017;30:327-343.

8. Lawitz E, Sulkowski MS, Ghalib R, et al. Simeprevir plus sofosbuvir, with or without ribavirin, to treat chronic infection with hepatitis $\mathrm{C}$ virus genotype 1 in non-responders to pegylated interferon and ribavirin and treatment-naive patients: the COSMOS randomised study. Lancet. 2014;384:1756-1765.

9. FDA approves combination simeprevir and sofosbuvir for HCV treatment. http://www.clinicaladvisor.com/web-exclusives/fda-approves-combination-simeprevir-andsofosbuvir-for-hcv-treatment/article/382126/. Accessed April 15, 2017.

10. European Medicines Agency. Sofosbuvir Approval. http://www.ema.europa.eu/ema/index.jsp?curl=pages/medicines/human/medicines/002798/hum an_med_001723.jsp\&mid=WC0b01ac058001d124. Accessed April 15, 2017.

11. European Medicines Agency. Simeprevir Approval. http://www.ema.europa.eu/ema/index.jsp?curl=pages/medicines/human/medicines/002777/hum an_med_001766.jsp\&mid=WC0b01ac058001d124. Accessed April 15, 2017.

12. Sulkowski MS, Vargas HE, Di Bisceglie AM, et al. Effectiveness of Simeprevir Plus Sofosbuvir, With or Without Ribavirin, in Real-World Patients With HCV Genotype 1 Infection. Gastroenterology. 2016;150:419-429.

13. Lutchman G, Nguyen NH, Chang CY, et al. Effectiveness and tolerability of simeprevir and sofosbuvir in nontransplant and post-liver transplant patients with hepatitis C genotype 1 . Aliment Pharmacol Ther. 2016;44:738-746. 
14. Dieterich D, Bacon B, Flamm SL, et al. Evaluation of sofosbuvir and simeprevir-based regimens in the TRIO network: academic and community treatment of a real-world, heterogeneous population. Hepatology. 2014;60(Suppl. 4):55A.

15. Butt AA, Yan P, Shaikh OS, Chung RT, Sherman KE. Sofosbuvir-based regimens in clinical practice achieve SVR rates closer to clinical trials: results from ERCHIVES. Liver Int. 2016;36:651-658.

16. Eletreby R, Elakel W, Said M, et al. Real life Egyptian experience of efficacy and safety of Simeprevir Sofosbuvir therapy in 6211 chronic HCV genotype IV infected patients. Liver Int. 2017;37:534-541.

17. Lawitz E, Matusow G, DeJesus E, et al. Simeprevir plus sofosbuvir in patients with chronic hepatitis $\mathrm{C}$ virus genotype 1 infection and cirrhosis: a phase 3 study (OPTIMIST-2). Hepatology. 2016;64:360-369.

18. Kwo P, Gitlin N, Nahass R, et al. Simeprevir plus sofosbuvir (12 and 8 weeks) in hepatitis C virus genotype 1-infected patients without cirrhosis: OPTIMIST-1, a phase 3 , randomized study. Hepatology. 2016;64:370-380.

19. EASL recommendations on treatment of hepatitis C 2016. J Hepatol. 2017;66:153-194.

20. EASL Recommendations on Treatment of Hepatitis C 2015. J Hepatol. 2015;63:199-236.

21. Guías AEEH/SEIMC de manejo de la Hepatitis C. www.aeeh.es. 2015. Accessed April 15, 2017.

22. EMA. Sofosbuvir Label of the Drug: Sovaldi (R).

23. EMA. Simeprevir Label of the Drug: Olysio(R).

24. European Medicines Agency. Ribavirin Label. http://www.ema.europa.eu/ema/index.jsp?curl=pages/medicines/human/medicines/001018/hum an_med_001037.jsp\&mid=WC0b01ac058001d124. Accessed April 15, 2017.

25. Llop E, Berzigotti A, Reig M, et al. Assessment of portal hypertension by transient elastography in patients with compensated cirrhosis and potentially resectable liver tumors. J Hepatol. 2012;56:103-108.

26. El-Khayat HR, Fouad YM, Maher M, El-Amin H, Muhammed H. Efficacy and safety of sofosbuvir plus simeprevir therapy in Egyptian patients with chronic hepatitis C: a real-world experience. Gut. 2016;1-5.

27. Raziky EI, Gamil M, Ashour MK, et al. Simeprevir plus sofosbuvir for eight or 12 weeks in treatment-naïve and treatment-experienced hepatitis $\mathrm{C}$ virus genotype 4 patients with or without cirrhosis. J Viral Hepat. 2017;24:102-111.

28. Gimeno-Ballester V, Simón MÁ, Trigo C, Mar J. San Miguel R. Sofosbuvir plus simeprevir for the treatment of Genotype 1 chronic hepatitis C: a review of evidence. Expert Rev. Gastroenterol Hepatol. 2016;10:1289-1303.

29. Colombo M, Russello M, Forte P, et al. Safety of Simeprevir-based treatment for hepatitis C in real practice: preliminary results from the stily observational study. J Hepatol. 2016;64:S796.

30. Pellicelli AM, Pace Palitti V, Vignally P, et al. Efficacy and safety of sofosbuvir/simeprevir plus flat dose ribavirin in genotype-1 elderly cirrhotic patients: a real life study. Liver Int. 2017;37:653-661.

31. Sarrazin C, Lathouwers E, Peeters M, et al. Prevalence of the hepatitis C virus NS3 polymorphism Q80K in genotype 1 patients in the European region. Antiviral Res. 2015;116:10-16.

32. Jimenez-Sousa MÁ, Gutiérrez-Rivas M, Álvaro-Meca A, et al. NS3 resistance-associated variants (RAVs) in patients infected with HCV genotype 1a in Spain. PLoS One. 2016;11:e0163197. https://doi.org/10.1371/journal.pone.0163197

33. Jacobson IM, Dore GJ, Foster GR, et al. Simeprevir with pegylated interferon alfa 2a plus ribavirin in treatment-naive patients with chronic hepatitis $\mathrm{C}$ virus genotype 1 infection (QUEST-1): a phase 3, randomised, double-blind, placebo-controlled trial. Lancet. 2014;384:403-413.

34. Forns X, Lawitz E, Zeuzem S, et al. Simeprevir with Peginterferon and Ribavirin leads to high rates of SVR in patients with HCV genotype 1 who relapsed after previous therapy: a phase 3 trial. Gastroenterology. 2014;146:1669-1679.

35. Thomson M, Konerman MA, Choxi H, Lok ASF. Primary Care Physician Perspectives on Hepatitis C Management in the Era of Direct-Acting Antiviral Therapy. Dig Dis Sci. 2016;61:3460-3468.

36. AASLD Recommendations for Testing, Managing, and Treating Hepatitis C. Hepatology. 2016. http://hcvguidelines.org/sites/default/files/HCV-Guidance_July_2016_b.pdf. Accessed April 15, 2017. 